\title{
Risk factors of postoperative recurrence and potential candidate of adjuvant radiotherapy in lung adenosquamous carcinoma
}

\author{
Jianjiao Ni ${ }^{1,2 \#}$, Zhiqin Zheng ${ }^{3 \#}$, Juan $\mathrm{Li}^{1,2}$, Yuan $\mathrm{Li}^{2,4}$, Min Fan ${ }^{1,2}$, Liang Liu ${ }^{1,2}$ \\ ${ }^{1}$ Department of Radiation Oncology, Fudan University Shanghai Cancer Center, Shanghai, China; ${ }^{2}$ Department of Oncology, Shanghai Medical \\ College, Fudan University, Shanghai, China; ${ }^{3}$ Department of Radiation Oncology, Fudan University Shanghai Cancer Center Minhang Branch \\ Hospital, Shanghai, China; ${ }^{4}$ Department of Pathology, Fudan University Shanghai Cancer Center, Shanghai, China \\ Contributions: (I) Conception and design: L Liu, M Fan; (II) Administrative support: M Fan; (III) Provision of study materials or patients: L Liu, M \\ Fan; (IV) Collection and assembly of data: J Ni, Z Zheng, J Li, Y Li; (V) Data analysis and interpretation: J Ni, Z Zheng; (VI) Manuscript writing: \\ All authors; (VII) Final approval of manuscript: All authors. \\ \#These authors contributed equally to this work. \\ Correspondence to: Liang Liu, MD; Min Fan, MD. Department of Radiation Oncology, Fudan University Shanghai Cancer Center, No. 270 Dongan \\ Road, Shanghai, China. Email: liuliangfudan@163.com; fanming@fudan.edu.cn.
}

Background: Adenosquamous carcinoma (ASC) is a rare and aggressive histologic subtype of non-small cell lung cancer (NSCLC). Little is known about the prognostic significance of routine immunohistochemical (IHC) markers and clinical value of adjuvant radiotherapy in completely resected lung ASC.

Methods: Consecutive patients with pathologically confirmed lung ASC receiving curative resection from January 2007 to December 2017 at our center were retrospectively reviewed. The prognostic significance of 14 routine IHC markers and potential candidate of adjuvant radiotherapy were investigated.

Results: With a median follow up of 35 (range, 3.0-138) months, 95 out of the 176 enrolled patients had disease recurrence. The 1-, 3- and 5-year cumulative rate of recurrence was $25.8 \%, 55.8 \%$ and $63.1 \%$, respectively. Using the Cox proportional hazard regression model, $\mathrm{T}$ stage, $\mathrm{N}$ stage, lymphovascular invasion (LVI), expression of CEA, expression of p53, but not EGFR mutations or expression of the other 12 IHC markers (CK20, CK5/6, PE10, ERCC1, Napsin A, RRM1, Ki67, CK7, P63, EGFR, HER2, TTF1), were significantly associated with postoperative recurrence. $\mathrm{N}$ stage, expression of CEA and LVI were identified as independent prognosticators of overall recurrence. Using competing risk methodology and distant recurrence chosen as a competing risk, $\mathrm{T}$ stage and $\mathrm{N}$ stage were identified as significant risk factors of locoregional recurrence. Moreover, adjuvant radiotherapy significantly improved disease-free survival (DFS) $(\mathrm{P}=0.002)$ and was associated with non-significant longer overall survival $(\mathrm{OS})(\mathrm{P}=0.078)$ among 95 patients with either pathological T3-4 or $\mathrm{N}^{+}$disease (collectively defined as $\mathrm{pT}_{3-4} / \mathrm{N}^{+}$disease).

Conclusions: This study provides the proof of concept for using routine IHC markers, along with common clinic-pathological parameters, in predicting postoperative recurrence and identifying potential candidate for adjuvant radiotherapy in completely resected lung ASC.

Keywords: Lung adenosquamous carcinoma (lung ASC); postoperative recurrence; radiotherapy

Submitted May 19, 2020. Accepted for publication Aug 28, 2020.

doi: $10.21037 /$ jtd-20-1979

View this article at: http://dx.doi.org/10.21037/jtd-20-1979

(c) Journal of Thoracic Disease. All rights reserved. 


\section{Introduction}

Lung adenosquamous carcinoma (ASC), consisting of histologic components of both squamous cell carcinoma and adenocarcinoma, with each component accounting for at least $10 \%$ of the tumor, comprises $0.4-4 \%$ of all lung cancers $(1,2)$. Several clinic-pathological variables have been found as prognostic biomarkers in surgically resected lung ASC, including pathological subtype, sex, and TNM stage $(3,4)$. However, the prognostic significance of routine immunohistochemical (IHC) markers, such as CEA, p53, CK20, CK5/6, PE10, ERCC1, Napsin A, RRM1, Ki67, CK7, P63, EGFR, HER2 and TTF1, remains elusive in lung ASC.

Lung ASC is reported to be more aggressive than the major types of non-small cell lung cancer (NSCLC) and postoperative recurrence poses a great challenge to surgeons and oncologists $(2,5)$. In order to optimize adjuvant therapy for completely resected lung ASC, the clinical patterns and risk factors of postoperative recurrence should be investigated (6). Additionally, the clinical value of adjuvant radiotherapy remain controversial. Postoperative radiotherapy has repeatedly been demonstrated to improve survival among patients with pN2 NSCLC in retrospective studies and large population cohorts, but has not been justified in prospective randomized clinical trials $(7-10)$. Whereas, the clinical value and potential candidate of adjuvant radiotherapy in lung ASC are seldom reported. Additionally, we presented the following article in accordance with the STROBE reporting checklist (11) (available at http://dx.doi.org/10.21037/jtd-20-1979).

\section{Methods}

\section{Patients}

Consecutive patients who underwent surgical resection with curative intention and had pathologically confirmed lung ASC from January 2007 to December 2017, were retrospectively enrolled. Exclusion criteria included neoadjuvant therapy, a second primary tumor, compromised resection, positive surgical margins, and death due to surgical complications. The main reason that patients receiving neoadjuvant therapy were excluded was neoadjuvant therapy may significantly alter the incidence and clinical patterns of postoperative recurrence. Our study followed The Declaration of Helsinki (as revised in 2013). The institutional review board of Fudan University Shanghai Cancer Center approved the study (approval number 090977-1). Informed consent was waived by the institutional review board because this was a retrospective study.

For each patient, common clinic-pathological parameters, including age, sex, tumor size, TNM stage, tumor differentiation, tumor histology, lymphovascular invasion (LVI), visceral pleural invasion (VPI) and EGFR mutation status, were gathered from electronic medical records. Pathologic TNM stage was in accordance with the eighth edition Lung Cancer Stage Classification (12). Tumor differentiation was determined on the basis of the 2015 World Health Organization Classification of Tumors of the Lung, Pleura, Thymus and Heart (13). Patients were divided into three pathological subtypes: adenocarcinomapredominant, squamous cell carcinoma-predominant and balanced, according to the criteria proposed by Gawrychowski (14). EGFR mutations were detected using the amplification-refractory mutation system (AMRS) method. Moreover, data on the expression of CEA, p53, CK20, CK5/6, PE10, ERCC1, Napsin A, RRM1, Ki67, CK7, P63, EGFR, HER2 and TTF1, were collected for each patient. IHC staining and evaluation were routinely performed in the Immunohistochemistry Diagnostic Laboratory of our cancer center.

\section{Treatment}

Curative surgery was carried out for each patient with systematic multilevel mediastinal lymph node dissection or adequate mediastinal sampling. Adjuvant radiotherapy was performed in line with our institutional protocol (15), using the intensity-modulated radiation therapy technique employing a linear accelerator with 6-MV X-rays. The total dose was generally about $50.4 \mathrm{~Gy}$, prescribed to the planning target volume and administered 1.8 Gy per fraction daily, 5 days per week. The decision of adjuvant radiotherapy was at the discretion of the treating physicians.

\section{Follow-up}

Follow up was at the discretion of the treating physicians, with chest computed tomography (CT) scans and ultrasonography of abdominal and cervical regions regularly performed, while brain magnetic resonance imaging (MRI) and bone scanning were not mandatory. In general, patients were followed up every 3 months in the first 2 years, every 6 months in the third to fifth years after surgery, and annually thereafter, in the clinic. Telephone follow-up calls 
were also implemented.

Postoperative recurrence was diagnosed considering all the evidence provided by CT, MRI, positron emission tomography-CT (PET-CT) scan or pathologic confirmation, and the initial sites of relapse were recorded. Postoperative recurrence developed in any site of the body was called overall recurrence. Postoperative recurrence was categorized into five groups according to the anatomic locations: thoracic recurrence, brain recurrence, neck recurrence, abdominal recurrence, and bone recurrence, which was called site-specific recurrence. The corresponding recurrences were simultaneously counted for patients who developed postoperative recurrence in more than one regions. Moreover, postoperative recurrence developed at the resection margin, the anastomosis, the mediastinal lymph nodes, or the ipsilateral hilar lymph nodes, were considered as loco-regional recurrence. Otherwise, it was defined as distant recurrence.

\section{Statistical analysis}

Disease-free survival (DFS) was calculated from the date of surgery to the documentation of initial postoperative recurrence or death due to any causes. Kaplan-Meier method was used to estimate survival, and differences among groups were investigated by the log-rank test. The prognosticators of overall recurrence were identified using the Cox proportional hazard regression model. The indicators of loco-regional recurrence were selected using competing risk methodology and Stata version 13.1 software (StataCorp, College Station, TX, USA). The time to site-specific recurrence or loco-regional recurrence, was censored by the time of documentation of postoperative recurrence, among patients who developed other types of recurrences first. Statistical analysis was performed using SPSS 21.0 (SPSS, Chicago, IL, USA). All assessment is considered to be significant when two-sided $\mathrm{P}$ value is less than 0.05 .

\section{Results}

\section{Patient characteristics}

A total of 176 patients were enrolled in this study and a flowchart for patient selection in this study was presented in Figure S1. Detailed disease characteristics of the enrolled patients, are illustrated in Table 1. Of note, EGFR mutations were detected in 97 patients, including 44 EGFR
Table 1 Disease characteristics

\begin{tabular}{|c|c|c|}
\hline Characteristics & Number $(n=176)$ & $\%$ \\
\hline \multicolumn{3}{|l|}{ Age } \\
\hline$\leq 60$ & 61 & 34.7 \\
\hline$>60$ & 115 & 65.3 \\
\hline \multicolumn{3}{|l|}{ Sex } \\
\hline Female & 62 & 36.0 \\
\hline Male & 114 & 64.0 \\
\hline \multicolumn{3}{|l|}{ Smoking history } \\
\hline Never & 77 & 43.8 \\
\hline Ever & 99 & 56.2 \\
\hline \multicolumn{3}{|l|}{ T stage } \\
\hline $\mathrm{T} 1$ & 85 & 48.3 \\
\hline $\mathrm{T} 2$ & 57 & 32.4 \\
\hline T3 & 23 & 13.1 \\
\hline T4 & 11 & 6.2 \\
\hline \multicolumn{3}{|l|}{$\mathrm{N}$ stage } \\
\hline NO & 97 & 55.1 \\
\hline $\mathrm{N} 1$ & 33 & 18.8 \\
\hline N2 & 46 & 26.1 \\
\hline \multicolumn{3}{|l|}{ TNM stage } \\
\hline I & 72 & 40.9 \\
\hline$\|$ & 40 & 22.7 \\
\hline III & 64 & 36.4 \\
\hline \multicolumn{3}{|l|}{ Pathological subtype } \\
\hline AC-predominant & 70 & 39.8 \\
\hline SCC-predominant & 67 & 38.1 \\
\hline Balanced & 39 & 22.1 \\
\hline \multicolumn{3}{|l|}{ EGFR mutation } \\
\hline WT & 79 & 44.9 \\
\hline MT & 97 & 55.1 \\
\hline \multicolumn{3}{|l|}{ LVI } \\
\hline Absent & 128 & 72.7 \\
\hline Present & 48 & 27.3 \\
\hline \multicolumn{3}{|l|}{ VPI } \\
\hline Absent & 143 & 81.2 \\
\hline Present & 33 & 18.8 \\
\hline \multicolumn{3}{|l|}{ PNI } \\
\hline Absent & 162 & 92.0 \\
\hline Present & 14 & 8.0 \\
\hline
\end{tabular}

AC, adenocarcinoma; SCC, squamous cell carcinoma; WT, wild type; MT, mutant; LVI, Iymphovascular invasion; VPI, visceral pleural invasion; $\mathrm{PNI}$, perineural invasion. 
A

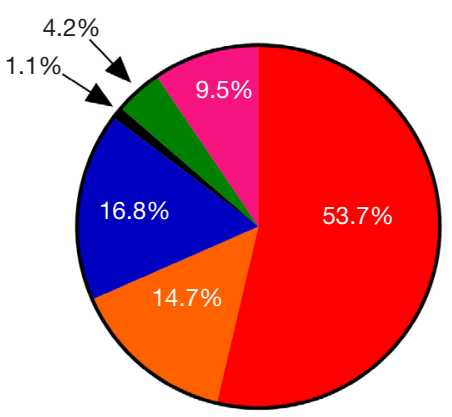

B

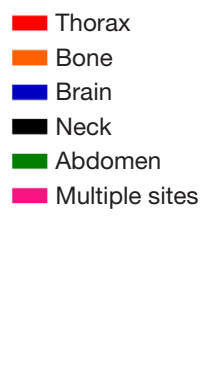

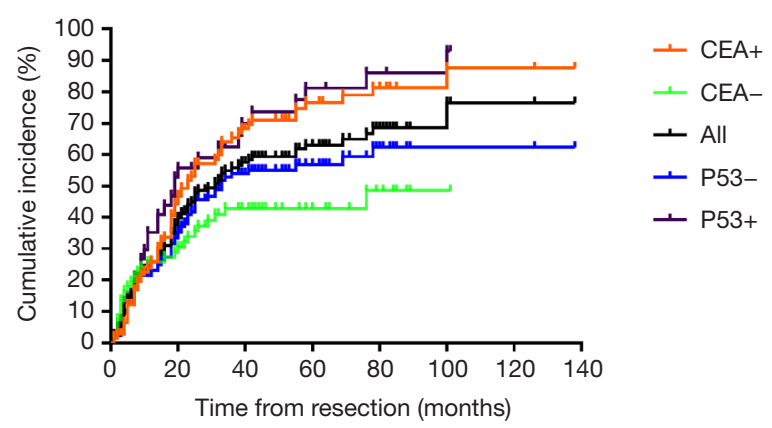

Figure 1 Patterns of initial recurrence in curative resected lung ASC. (A) Pie chart demonstrating the distribution of initial recurrence site; (B) cumulative incidence of postoperative recurrence in the whole population (all) or stratified by the expression status of CEA and p53. ASC, adenosquamous carcinoma.

L858R mutations, 49 EGFR exon 19 deletions, 1 EGFR G719X mutation, 1 EGFR T790M mutation and 2 EGFR exon 20 insertions.

In addition, the positive rate of CEA, p53, CK20, CK5/6, PE10, ERCC1, Napsin A, RRM1, CK7, P63, EGFR, HER2 and TTF1, was $64.2 \%, 78.9 \%, 9.5 \%, 71.2 \%, 45.8 \%$, $64.9 \%, 68.9 \%, 81.7 \%, 89.7 \%, 81.7 \%, 77.1 \%, 41.4 \%$ and $77.1 \%$, respectively. The expression of Ki67 lower than $50 \%$ was detected in 107 (60.8\%) patients, and no less than $50 \%$ was detected in the rest $69(39.2 \%)$ patients.

\section{Patterns of postoperative recurrence}

Post-surgery, 110 patients received adjuvant systematic therapy, consisting of chemotherapy in 101 patients, EGFR targeted therapy in seven patients, a combination of chemotherapy and EGFR targeted therapy in two patients. Besides, 35 patients received adjuvant radiotherapy according to the institutional guideline developed in 2004 (15). In addition, 63 patients did not receive any adjuvant treatment. With a median follow up of 35.0 (range, 3.0-138.0) months, disease recurrence occurred in 95 patients. The 1-, 3-, 5- and 10-year cumulative rate of recurrence was $25.8 \%, 55.8 \%, 63.1 \%$ and $76.5 \%$, respectively.

The pattern of postoperative recurrence was displayed in Figure $1 A$, and the frequencies of initial recurrence in the thorax, brain, bone, abdomen and neck, were $53.7 \%$, $16.8 \%, 14.7 \%, 4.2 \%$ and $1.1 \%$, respectively. In addition, nine patients developed their initial relapse in more than one sites simultaneously. In terms of loco-regional recurrence, initial recurrence at the anastomosis of the surgery was observed in four patients and initial relapse in the mediastinal or hilar lymph nodes was observed in 14 patients.

\section{Prognosticators of postoperative recurrence}

Univariate Cox regression analyses revealed that $\mathrm{T}$ stage, $\mathrm{N}$ stage, LVI, as well as expression of p53 and CEA, were significantly associated with overall recurrence. Of note, EGFR mutations was not associated with overall recurrence $(\mathrm{P}=0.824)$. Multivariate Cox regression analyses identified $\mathrm{N}$ stage, LVI and expression of CEA as independent prognosticators (Table 2). Specifically, among patients with positive expression of CEA, the 1-, 3-, 5- and 10-year cumulative rate of recurrence were $26.0 \%, 65.4 \%, 76.6 \%$ and $87.5 \%$, respectively. Conversely, among patients with negative expression of CEA, the 1-, 3-, 5- and 10-year cumulative rate of recurrence were $25.8 \%, 42.8 \%, 42.8 \%$ and $48.5 \%$, respectively. On the other hand, among patients with negative expression of p53, the 1-, 3-, 5- and 10 -year cumulative rate of recurrence were $23.2 \%, 53.9 \%$, $56.8 \%$ and $62.3 \%$, respectively. While, among patients with positive expression of $\mathrm{p} 53$, the 1-, 3-, 5- and 10-year cumulative rate of recurrence were $35.3 \%, 62.5 \%, 81.2 \%$ and $93.0 \%$, respectively (Figure $1 B$ ).

Furthermore, using distant recurrence as a competing risk, T stage (T3-4 vs. T1-2) and $\mathrm{N}$ stage (N1-2 vs. N0), were found to be significantly associated with higher risk of loco-regional recurrence, with a hazard ratio (HR) of 1.402 [95\% confidential interval (CI): $1.237-2.438, \mathrm{P}=0.042$ ] and 2.871 (95\% CI: 1.8353-4.197, P=0.038), respectively. None of the other clinic-pathological parameters or IHC markers 
Table 2 Cox proportional hazard analyses of clinic-pathological parameters and IHC markers associated with overall recurrence

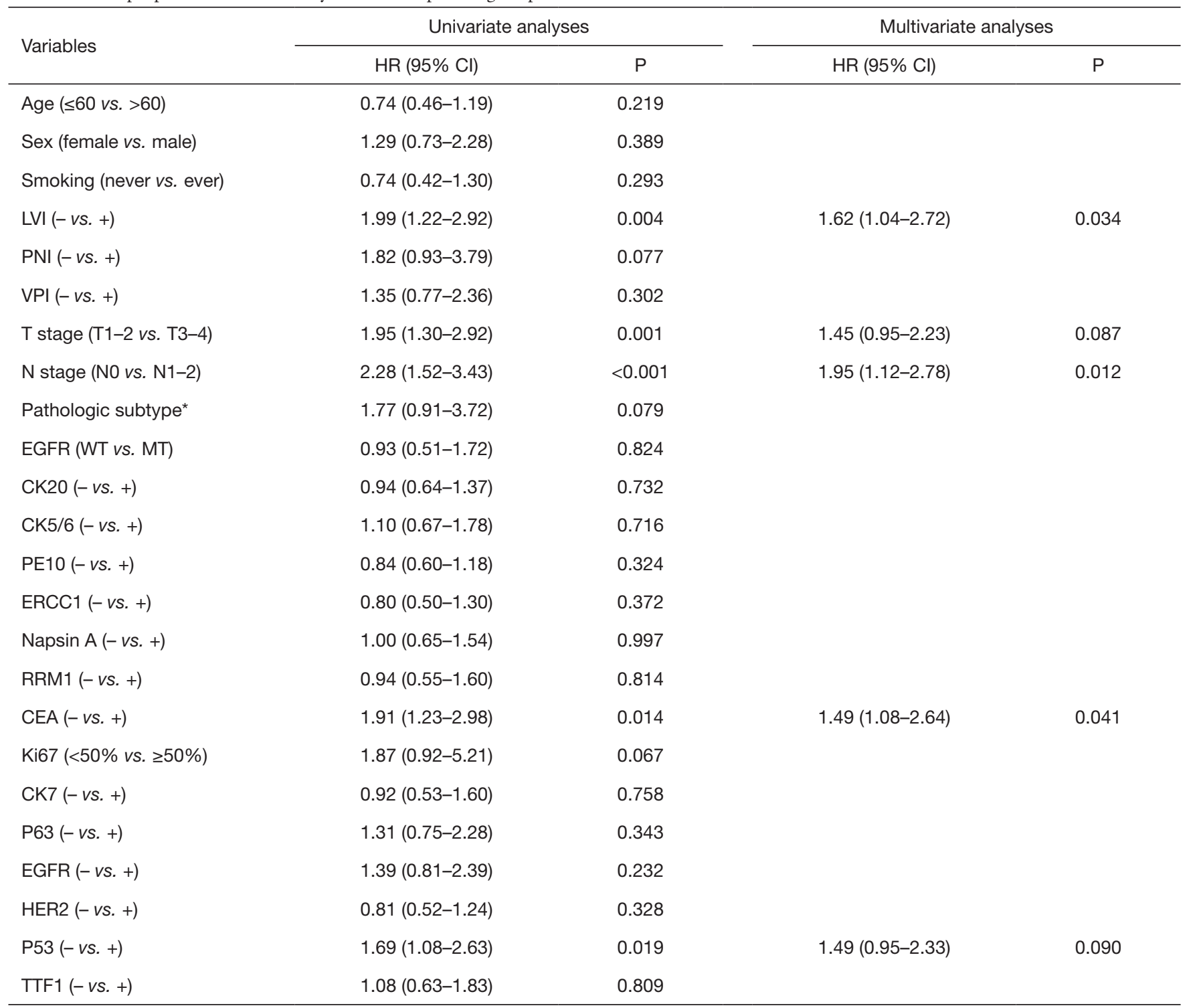

*, Balanced vs. non-balanced (adenocarcinoma- or squamous cell carcinoma-predominant). HR, hazard ratio; Cl, confidential interval; LVI, lymphovascular invasion; PNI, perineural invasion; VPI, visceral pleural invasion; WT, wild type; MT, mutant.

listed in Table 2 were demonstrated to be significantly associated with loco-regional recurrence.

\section{Clinical value of adjuvant radiotherapy}

As has been mentioned above, advanced T stage (T3-4) and $\mathrm{N}$ stage $\left(\mathrm{N}^{+}\right)$were significantly associated with loco-regional recurrence, we set out to investigate the clinical value of adjuvant radiotherapy in patients with either $\mathrm{T} 3-4$ disease or $\mathrm{N}^{+}$disease (defined collectively as $\mathrm{pT}_{3-4} / \mathrm{N}^{+}$). There were 95 patients with $\mathrm{pT}_{3-4} / \mathrm{N}^{+}$disease in this study and the baseline disease characteristics of those received adjuvant radiotherapy $(\mathrm{n}=33)$ and those $\operatorname{did} \operatorname{not}(\mathrm{n}=62)$ were generally balanced (Table S1). Among these 95 patients, adjuvant radiotherapy was associated with improved DFS (16.0 vs. 34.0 months, $\mathrm{P}=0.002$ ) (Figure $2 A$ ) and non-significantly longer overall survival (OS) (Figure 2B).

By the time of data cut off, 48 patients had died and the 1-, 3-, 5- and 10-year OS were 92.7\% (95\% CI: 90.7-94.7\%), 74.8\% (95\% CI: 71.0-78.6\%), 65.9\% (95\% 

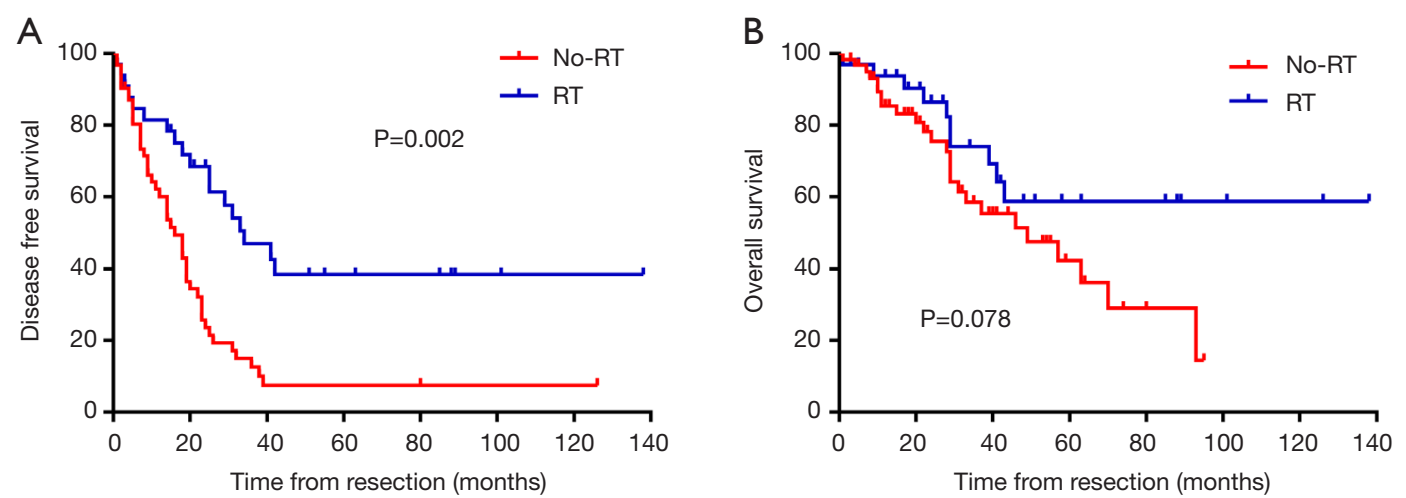

Figure 2 Prognostic significance of adjuvant radiotherapy in lung ASC. Kaplan-Meier survival curve of DFS (A) and OS (B) stratified by the status of adjuvant RT among patients with $\mathrm{pT}_{3-4} / \mathrm{N}^{+}$disease. ASC, adenosquamous carcinoma; DFS, disease-free survival; OS, overall survival; RT, radiotherapy.

CI: $61.4-70.4 \%$ ) and $48.3 \%$ (95\% CI: $40.1-56.5 \%$ ), respectively. Univariate Cox regression analyses discovered that $\mathrm{T}$ stage, $\mathrm{N}$ stage and LVI were significantly associated with OS. Multivariate Cox regression analyses demonstrated that only $\mathrm{N}$ stage was an independent predictor of OS (Table 3).

\section{Discussion}

Previous investigations have identified several prognostic biomarkers in surgically resected lung ASC $(2-4,6)$, but few studies have examined the prognostic significance of routine IHC markers and the clinical value of adjuvant radiotherapy in completely resected lung ASC. To the best of our knowledge, this is the first study incorporating common clinic-pathological parameters and a large panel of routine IHC markers to predict postoperative recurrence and identify potential candidate for adjuvant radiotherapy in lung ASC.

Recent studies have shed light on the prognostic values of routine IHC markers in lung cancer (16-19) and our study demonstrated that the expression status of CEA and p53 may serve as powerful biomarkers in lung ASC. Expression of CEA is shown to be associated with shorter survival in colorectal cancer (20) and the prognostic significance of serum CEA in NSCLC has been repeatedly demonstrated (21-23). In addition, expression of $\mathrm{p} 53$ is found to be prognostic in various human cancers, such as breast cancer (24), NSCLC (19), gastric cancer (25), bladder cancer (26) and cervical adenocarcinoma (27). In the current study, expression of CEA and p53, along with
T stage, $\mathrm{N}$ stage and LVI, were found to be significantly associated with postoperative recurrence in lung ASC, which were generally consistent with previous studies. All these five clinic-pathological variables are easy to collect from routine clinical practice, which could facilitate the process of decision-making for treating physicians.

The prognostic significance of EGFR mutations in NSCLC remain controversial and it has not been specifically examined in lung ASC. In the current study, EGFR mutations were detected in $55.1 \%$ of patients, the frequency of which was comparable to previous studies conducted in Asian populations (28-30). Of note, among patients with non-squamous NSCLC and lung ASC, EGFR mutations are detected in a similar rate and EGFR tyrosine kinase inhibitors are reported to induce similar efficacy $(31,32)$. Moreover, EGFR mutations are reported to be detected in both components of squamous cell carcinoma and adenocarcinoma from the same lung ASC patient (2). All of these support the hypothesis that the two different histological components of lung ASC originate from common precursors (2,33). Additionally, a recent meta-analysis including 19 relevant studies and a total of 4,872 surgically resected NSCLC patients found that EGFR mutations was not associated with DFS (34). Moreover, a Japanese study including 32 lung ASC patients demonstrated that EGFR mutations were not associated with recurrence-free survival or OS (35), which was reproduced by our study with a larger sample size.

Other common prognostic parameters, such as pathological subtype and sex, are reported to be prognostic in surgically resected lung ASC, which however were 
Table 3 Cox proportional hazard analyses of clinic-pathological parameters and IHC markers associated with OS

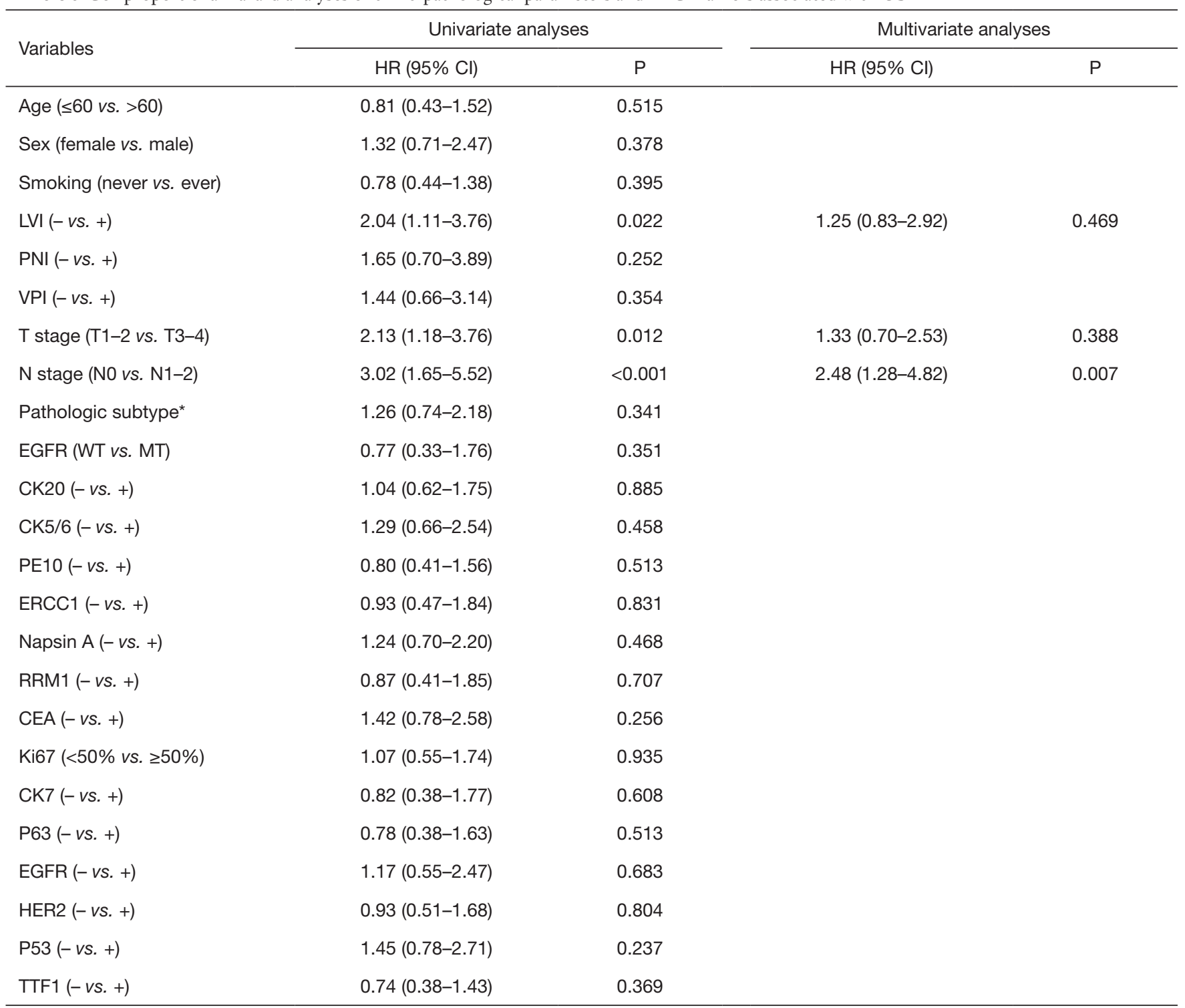

*, Balanced vs. non-balanced (adenocarcinoma- or squamous cell carcinoma-predominant). OS, overall survival; HR, hazard ratio; Cl, confidential interval; LVI, lymphovascular invasion; PNI, perineural invasion; VPI, visceral pleural invasion; WT, wild type; MT, mutant.

not reproduced in our study. Pathological subtype was repeatedly found to be an important prognostic factor among surgically resected lung ASC $(3,4,14)$. However, the results were conflicting, as patients with balanced subtype were demonstrated to have superior prognosis in two of the studies $(4,14)$, but were revealed to have worse survival than those with squamous cell carcinoma-predominant subtype in another study (3). In the current study, there was a trend toward better prognosis of balanced subtype compared with the other two pathological subtypes, however the different did not reach statistical significance $(\mathrm{P}=0.079)$. Larger prospective studies including patients treated with modern surgical techniques and cutting-edge biological therapies (such as targeted therapy and immune checkpoint inhibitors) are needed to further examined the prognostic role of pathological subtypes. Similarly, sex was found to be prognostic in surgically resected lung ASC (3), but was not reproduced by other studies $(4,6,14)$ as well as our study. As a retrospective study, we could not gather the information on the mutation status of several other common driver 
mutations in NSCLC, such as ALK, ROS1, KRAS and RET. The prognostic significance of these molecular aberrations remain unknown in surgically resected lung ASC.

The clinical value of adjuvant radiotherapy in lung ASC has not been fully investigated and our study identified subgroup of patients who may benefit from adjuvant radiotherapy. Postoperative radiotherapy has been suggested to be beneficial for stage III/N2 NSCLC $(7,8)$ and previous studies from our center had demonstrated similar results $(15,36)$. However, the clinical value of adjuvant radiotherapy in lung ASC is seldom reported. Since accumulating data indicate that lung ASC is more aggressive than common NSCLC $(2,5,6)$, we hypothesized that adjuvant radiotherapy may provide survival benefit in a larger subset of patients with lung ASC. In our study, advanced $\mathrm{T}$ stage (T3-4) and $\mathrm{N}$ stage $\left(\mathrm{N}^{+}\right)$were found to be significantly associated with loco-regional recurrence, which may otherwise be prevented by postoperative radiotherapy. Hence, we set out to investigate the clinical value of adjuvant radiotherapy among patients with $\mathrm{pT}_{3-4} / \mathrm{N}^{+}$disease. Indeed, adjuvant radiotherapy significantly improved DFS and tended to prolong OS for patients with $\mathrm{pT}_{3-4} / \mathrm{N}^{+}$lung ASC in our study. Of note, all of the patients with $\mathrm{pT}_{3-4} / \mathrm{N}^{+}$disease received adjuvant systematic therapy and the baseline disease characteristics between patients who received adjuvant radiotherapy and those who didn't were generally well balanced. To the best of our knowledge, this is the first study to identify potential candidate for adjuvant radiotherapy in lung ASC, which need to be verified in larger prospective studies.

Our study also has some limitations. Due to the nature of retrospective study, the clinical values of adjuvant radiotherapy need to be interpreted with caution, as certain selection bias may exist. Second, postoperative follow-ups were generally at the discretion of the treating physicians and patients with asymptomatic recurrence may be underestimated, especially for those with brain and/or bone metastases, as brain MRI and bone scanning were not mandatory.

In conclusion, our study indicates that expression status of CEA and p53 are powerful biomarkers in curatively resected lung ASC. Adjuvant radiotherapy provided survival benefit among $\mathrm{pT}_{3-4} / \mathrm{N}^{+}$lung ASC.

\section{Acknowledgments}

We thank Edanz Editing China (www.liwenbianji.cn/ac) for editing the English text of a draft of this manuscript.
Funding: This work was supported by the National Natural Science Foundation of China (No. 81802904 to LL) and the Fund of Fudan University Shanghai Cancer Center (No. YJ201713 to LL).

\section{Footnote}

Reporting Checklist: The authors have completed the STROBE reporting checklist. Available at http://dx.doi. org/10.21037/jtd-20-1979

Data Sharing Statement: Available at http://dx.doi. org/10.21037/jtd-20-1979

Conflicts of Interest: All authors have completed the ICMJE uniform disclosure form (available at http://dx.doi. org/10.21037/jtd-20-1979). The authors have no conflicts of interest to declare.

Ethical Statement: The authors are accountable for all aspects of the work in ensuring that questions related to the accuracy or integrity of any part of the work are appropriately investigated and resolved. Our study followed The Declaration of Helsinki (as revised in 2013). The institutional review board of Fudan University Shanghai Cancer Center approved the study (approval number 090977-1). Informed consent was waived by the institutional review board because this was a retrospective study.

Open Access Statement: This is an Open Access article distributed in accordance with the Creative Commons Attribution-NonCommercial-NoDerivs 4.0 International License (CC BY-NC-ND 4.0), which permits the noncommercial replication and distribution of the article with the strict proviso that no changes or edits are made and the original work is properly cited (including links to both the formal publication through the relevant DOI and the license). See: https://creativecommons.org/licenses/by-nc-nd/4.0/.

\section{References}

1. Cooke DT, Nguyen DV, Yang Y, et al. Survival comparison of adenosquamous, squamous cell, and adenocarcinoma of the lung after lobectomy. Ann Thorac Surg 2010;90:943-8.

2. Wang J, Wang $\mathrm{Y}$, Tong $\mathrm{M}$, et al. Research progress of the clinicopathologic features of lung adenosquamous carcinoma. Onco Targets Ther 2018;11:7011-7. 
3. Zhu L, Jiang L, Yang J, et al. Clinical characteristics and prognosis of patients with lung adenosquamous carcinoma after surgical resection: results from two institutes. J Thorac Dis 2018;10:2397-402.

4. Zhao H, Yang H, Yao F, et al. Improved survival associated with a balanced structure between adenomatous and squamous components in patients with adenosquamous carcinoma of the lung. Eur J Surg Oncol 2016;42:1699-706.

5. Nakagawa K, Yasumitu T, Fukuhara K, et al. Poor prognosis after lung resection for patients with adenosquamous carcinoma of the lung. Ann Thorac Surg 2003;75:1740-4.

6. Mordant P, Grand B, Cazes A, et al. Adenosquamous carcinoma of the lung: surgical management, pathologic characteristics, and prognostic implications. Ann Thorac Surg 2013;95:1189-95.

7. Mikell JL, Gillespie TW, Hall WA, et al. Postoperative radiotherapy is associated with better survival in non-small cell lung cancer with involved N2 lymph nodes: results of an analysis of the National Cancer Data Base. J Thorac Oncol 2015;10:462-71.

8. Herskovic A, Mauer E, Christos P, et al. Role of postoperative radiotherapy in pathologic stage IIIA (N2) non-small cell lung cancer in a prospective nationwide oncology outcomes database. J Thorac Oncol 2017;12:302-13.

9. Shen WY, Ji J, Zuo YS, et al. Comparison of efficacy for postoperative chemotherapy and concurrent radiochemotherapy in patients with IIIA-pN2 non-small cell lung cancer: an early closed randomized controlled trial. Radiother Oncol 2014;110:120-5.

10. Sun JM, Noh JM, Oh D, et al. Randomized phase II trial comparing chemoradiotherapy with chemotherapy for completely resected unsuspected N2-positive non-small cell lung cancer. J Thorac Oncol 2017;12:1806-13.

11. von Elm E, Altman DG, Egger M, et al. The Strengthening the Reporting of Observational Studies in Epidemiology (STROBE) statement: guidelines for reporting observational studies. PLoS Med 2007;4:e296.

12. Detterbeck FC, Boffa DJ, Kim AW, et al. The eighth edition lung cancer stage classification. Chest 2017;151:193-203.

13. Travis WD, Brambilla E, Nicholson AG, et al. The 2015 World Health Organization Classification of lung tumors: impact of genetic, clinical and radiologic advances since the 2004 classification. J Thorac Oncol 2015;10:1243-60.

14. Gawrychowski J, Brulinski K, Malinowski E, et al.
Prognosis and survival after radical resection of primary adenosquamous lung carcinoma. Eur J Cardiothorac Surg 2005;27:686-92.

15. Feng $W, F u$ XL, Cai XW, et al. Patterns of local-regional failure in completely resected stage $\mathrm{IIIA}(\mathrm{N} 2)$ non-small cell lung cancer cases: implications for postoperative radiation therapy clinical target volume design. Int J Radiat Oncol Biol Phys 2014;88:1100-7.

16. Komaki R, Paulus R, Blumenschein GR Jr, et al. EGFR expression and survival in patients given cetuximab and chemoradiation for stage III non-small cell lung cancer: a secondary analysis of RTOG 0324 . Radiother Oncol 2014;112:30-6.

17. Kasajima A, Konukiewitz B, Oka N, et al. Clinicopathological profiling of lung carcinoids with a Ki67 index > 20. Neuroendocrinology 2019;108:109-20.

18. Vilmar AC, Santoni-Rugiu E, Sorensen JB. Predictive impact of RRM1 protein expression on vinorelbine efficacy in NSCLC patients randomly assigned in a chemotherapy phase III trial. Ann Oncol 2013;24:309-14.

19. Graziano SL, Gu L, Wang X, et al. Prognostic significance of mucin and p53 expression in stage IB non-small cell lung cancer: a laboratory companion study to CALGB 9633. J Thorac Oncol 2010;5:810-7.

20. Ishida H, Miwa H, Tatsuta M, et al. Ki-67 and CEA expression as prognostic markers in Dukes' C colorectal cancer. Cancer Lett 2004;207:109-15.

21. Muley T, Rolny V, He Y, et al. The combination of the blood based tumor biomarkers cytokeratin 19 fragments (CYFRA 21-1) and carcinoembryonic antigen (CEA) as a potential predictor of benefit from adjuvant chemotherapy in early stage squamous cell carcinoma of the lung (SCC). Lung Cancer 2018;120:46-53.

22. Dehing-Oberije C, Aerts H, Yu S, et al. Development and validation of a prognostic model using blood biomarker information for prediction of survival of non-small-cell lung cancer patients treated with combined chemotherapy and radiation or radiotherapy alone (NCT00181519, NCT00573040, and NCT00572325). Int J Radiat Oncol Biol Phys 2011;81:360-8.

23. Grunnet M, Sorensen JB. Carcinoembryonic antigen (CEA) as tumor marker in lung cancer. Lung Cancer 2012;76:138-43.

24. Millar EK, Graham PH, McNeil CM, et al. Prediction of outcome of early ER+ breast cancer is improved using a biomarker panel, which includes Ki-67 and p53. Br J Cancer 2011;105:272-80.

25. Deveci MS, Deveci G. Prognostic value of $\mathrm{p} 53$ protein and 
MK-1 (a tumor-associated antigen) expression in gastric carcinoma. Gastric Cancer 2007;10:112-6.

26. Garcia del Muro X, Condom E, Vigues F, et al. p53 and p21 Expression levels predict organ preservation and survival in invasive bladder carcinoma treated with a combined-modality approach. Cancer 2004;100:1859-67.

27. Suzuki $Y$, Nakano T, Kato S, et al. Immunohistochemical study of cell cycle-associated proteins in adenocarcinoma of the uterine cervix treated with radiotherapy alone: P53 status has a strong impact on prognosis. Int J Radiat Oncol Biol Phys 2004;60:231-6.

28. Jia XL, Chen G. EGFR and KRAS mutations in Chinese patients with adenosquamous carcinoma of the lung. Lung Cancer 2011;74:396-400.

29. Shi $X, W u H, L u J$, et al. Screening for major driver oncogene alterations in adenosquamous lung carcinoma using PCR coupled with next-generation and Sanger sequencing methods. Sci Rep 2016;6:22297.

30. Wang R, Pan Y, Li C, et al. Analysis of major known driver mutations and prognosis in resected adenosquamous lung carcinomas. J Thorac Oncol 2014;9:760-8.

31. Fan L, Yang H, Yao F, et al. Clinical outcomes of epidermal growth factor receptor tyrosine kinase inhibitors in recurrent adenosquamous carcinoma of the lung after resection. Onco Targets Ther 2017;10:239-45.

32. Zhang C, Yang H, Lang B, et al. Surgical significance and efficacy of epidermal growth factor receptor tyrosine kinase inhibitors in patients with primary lung adenosquamous carcinoma. Cancer Manag Res 2018;10:2401-7.

33. Gerlinger M, Rowan AJ, Horswell S, et al. Intratumor heterogeneity and branched evolution revealed by multiregion sequencing. N Engl J Med 2012;366:883-92.

34. He Q, Xin P, Zhang M, et al. The impact of epidermal growth factor receptor mutations on the prognosis of resected non-small cell lung cancer: a meta-analysis of literatures. Transl Lung Cancer Res 2019;8:124-34.

35. Morodomi Y, Okamoto T, Takenoyama M, et al. Clinical significance of detecting somatic gene mutations in surgically resected adenosquamous cell carcinoma of the lung in Japanese patients. Ann Surg Oncol 2015;22:2593-8.

36. Yuan C, Tao X, Zheng D, et al. The lymph node status and histologic subtypes influenced the effect of postoperative radiotherapy on patients with $\mathrm{N} 2$ positive IIIA non-small cell lung cancer. J Surg Oncol 2019;119:379-87.
Cite this article as: $\mathrm{Ni} \mathrm{J}$, Zheng Z, Li J, Li Y, Fan M, Liu L. Risk factors of postoperative recurrence and potential candidate of adjuvant radiotherapy in lung adenosquamous carcinoma. J Thorac Dis 2020;12(10):5593-5602. doi: 10.21037/jtd-20-1979 
Patients who had curative resection and confirmed adenosquamous carcinoma from January 2007 to December 2017 at FUSCC $(n=208)$

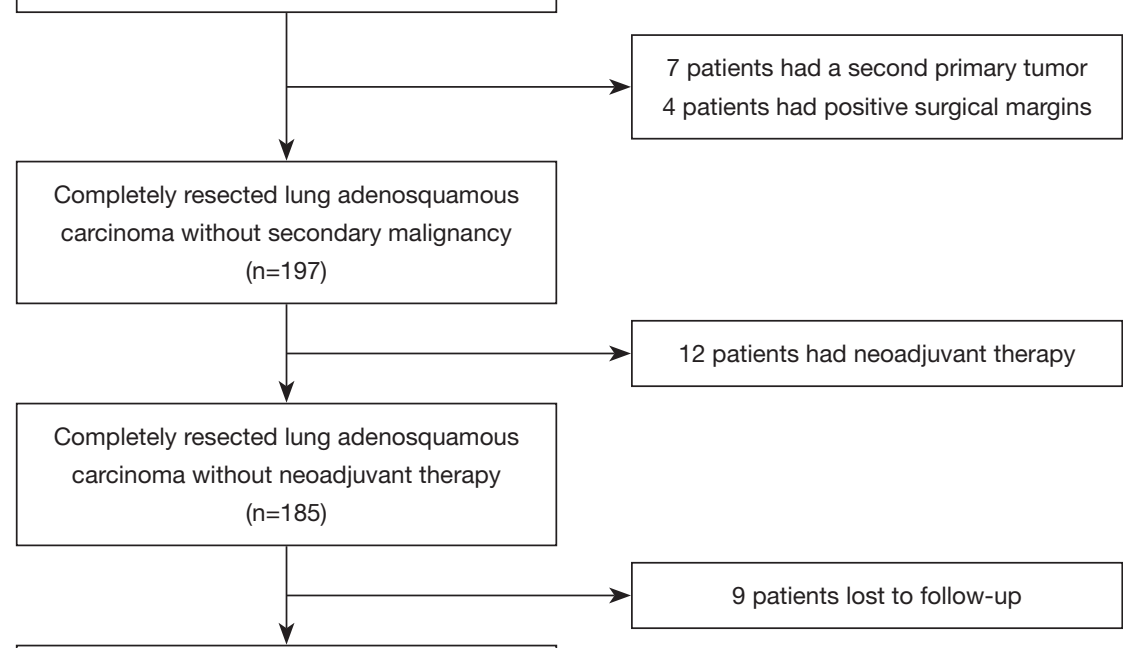

Patients finally enrolled $(n=176)$

Figure S1 Flowchart of patient enrollment. FUSCC, Fudan University Shanghai Cancer Center. 
Table S1 Disease characteristics of $\mathrm{pT}_{3-4} / \mathrm{N}^{+}$lung ASC $(\mathrm{n}=95)$

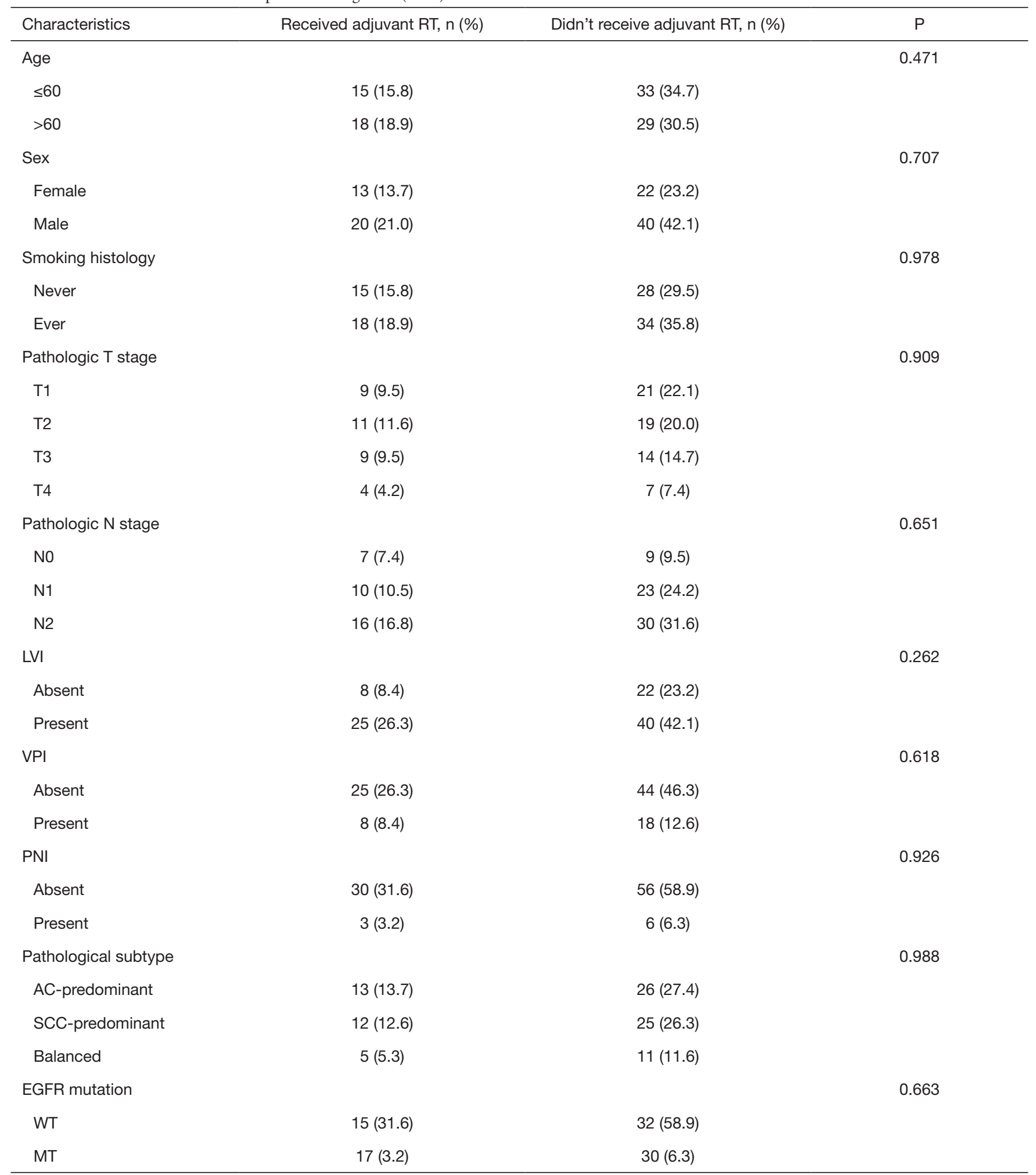

RT, radiotherapy; LVI, lymphovascular invasion; VPI, visceral pleural invasion; PNI, perineural invasion; AC, adenocarcinoma; SCC, squamous cell carcinoma; WT, wild type; MT, mutant. 